\title{
Anomalous Josephson Effect between Even- and Odd-Frequency Superconductors
}

\author{
Yukio Tanaka, ${ }^{1,2}$ Alexander A. Golubov, ${ }^{3}$ Satoshi Kashiwaya, ${ }^{4}$ and Masahito Ueda ${ }^{5}$ \\ ${ }^{1}$ Department of Applied Physics, Nagoya University, Nagoya 464-8603, Japan \\ ${ }^{2}$ CREST Japan Science and Technology Cooperation (JST), Nagoya 464-8603, Japan \\ ${ }^{3}$ Faculty of Science and Technology, University of Twente, Enschede, 7500 AE, The Netherlands \\ ${ }^{4}$ National Institute of Advanced Industrial Science and Technology (AIST), Tsukuba 305-8568, Japan \\ ${ }^{5}$ Department of Physics, Tokyo Institute of Technology, Tokyo 152-8551, Japan \\ (Received 1 October 2006; revised manuscript received 27 December 2006; published 20 July 2007)
}

\begin{abstract}
We demonstrate that, contrary to standard wisdom, the lowest-order Josephson coupling is possible between odd- and even-frequency superconductors. The origin of this effect is the induced odd- (even)frequency pairing component at the interface of bulk even- (odd-)frequency superconductors. The resulting current-phase relation is found to be proportional to $\cos \varphi$, where $\varphi$ is the macroscopic phase difference between the two superconductors.
\end{abstract}

DOI: 10.1103/PhysRevLett.99.037005

It is well known that Josephson coupling occurs between superconductors with the same order parameter symmetry [1]. Generally, symmetries with respect to momentum, spin, and time are to be considered. According to symmetry with respect to time, superconductors are classified into even-frequency and odd-frequency superconductors. In accordance with the Fermi-Dirac statistics, even-frequency superconductors are characterized by the spin-singlet evenparity or spin-triplet odd-parity pairing state, while oddfrequency superconductors feature the spin-singlet oddparity or spin-triplet even-parity pairing state. It has been suggested in Ref. [2] from basic symmetry arguments that the lowest-order Josephson coupling should be absent between odd- and even-frequency superconductors. However, as shown in the present Letter, the Josephson current can flow via an interface-induced state.

The underlying physics behind this phenomenon can be explained as follows. Near the interface, due to the breakdown of translational invariance, the pair potential acquires a spatial dependence which leads to coupling between the even- and odd-parity pairing states. The Fermi-Dirac statistics then dictates that the induced pair amplitude at the interface should be odd (even) in frequency when the bulk pair potential has an even- (odd-)frequency component. Consequently, the Josephson coupling between even- and odd-frequency superconductors becomes possible. To be compatible with the time reversal invariance in each superconductor, the phase of the interface-induced pair amplitude undergoes a $\pi / 2$ shift from that of the bulk one. This twist of the phase of the pair amplitude gives rise to an anomalous Josephson current, whereby the current phase relation is proportional to $\cos \varphi$. Although both superconductors do not break the time reversal symmetry themselves, the resulting Josephson coupling does break this symmetry, since the parities with respect to frequency dependence in even- and odd-frequency superconductors differ from each other.

Up to now, almost all of the known superconductors belong to the symmetry class of the even-frequency pair-
PACS numbers: 74.45.+c, 74.20.Rp, 74.50.+r

ing. The possibility of the odd-frequency pairing state in a uniform system was proposed for ${ }^{3} \mathrm{He}$ in Ref. [3] and for a superconducting state involving strong correlations in Refs. [2,4-6]. The realization of the odd-frequency pairing state in inhomogeneous systems has recently been proposed in ferromagnet-superconductor heterostructures [7]. Furthermore, two of the present authors have predicted that the odd-frequency pairing state can be induced in a diffusive normal metal attached to a spin-triplet superconductor [8]. However, in these examples the odd-frequency pairing is realized due to spin-triplet correlations. A question naturally arises as to whether the spin-triplet ordering is a necessary condition for the existence of the oddfrequency pairing state at a superconducting interface.

In this Letter we address the issue of odd-frequency pairing in the generic case of a normal metalsuperconductor $(N / S)$ interface, where $S$ has an evenfrequency pairing state in the bulk. We will show that, quite generally, the odd-frequency component is induced near a superconducting interface due to the spatial variation of the pair potential. If a superconductor has an evenfrequency spin-singlet even-parity (ESE) or evenfrequency spin-triplet odd-parity (ETO) pairing state in the bulk, the pair amplitude at the interface possesses, respectively, odd-frequency spin-singlet odd-parity (OSO) or odd-frequency spin-triplet even-parity (OTE). Similarly, in the case of a bulk odd-frequency superconductor, an even-frequency component of the pair amplitude is induced at the interface. An important application of these results is the existence of Josephson coupling between bulk odd- and even-frequency superconductors to the lowest order in transmission coefficient $T_{m}$. The resulting current-phase relation is proportional to $\cos \varphi$, in contrast to the conventional $\sin \varphi$ dependence.

In the following analysis, we consider an $N / S$ junction as the simplest example of a nonuniform superconducting system without impurity scattering. Both the ESE and ETO states are considered in the superconductor. As regards the spin-triplet superconductor, we choose $S_{z}=0$ for simplic- 
ity. We assume a thin insulating barrier located at the $N / S$ interface $(x=0)$ with $N(x<0)$ and $S(x>0)$ modeled by delta function $H \delta(x)$, where $H$ is the magnitude of the strength of the delta function potential. The reflection coefficient of the junction for the quasiparticle for injection angle $\theta$ is given by $R=Z^{2} /\left(Z^{2}+4 \cos ^{2} \theta\right)=1-T_{m}$ with $Z=2 H / v_{F}$, where $\theta(-\pi / 2<\theta<\pi / 2)$ is measured from the normal to the interface and $v_{F}$ is the Fermi velocity. The quasiclassical Green's function in a superconductor is parametrized in terms of

$$
\hat{g}_{ \pm}=f_{1 \pm} \hat{\tau}_{1}+f_{2 \pm} \hat{\tau}_{2}+g_{ \pm} \hat{\tau}_{3}, \quad \hat{g}_{ \pm}^{2}=\hat{1}
$$

with Pauli matrices $\hat{\tau}_{i}(i=1-3)$ and unit matrix $\hat{1}$. Here, the index $+(-)$ denotes left- (right-)going quasiparticles [9]. It is possible to express the above anomalous Green's function as

$$
f_{1 \pm}=\frac{ \pm i\left(F_{ \pm}+D_{ \pm}\right)}{1-D_{ \pm} F_{ \pm}}, \quad f_{2 \pm}=\frac{D_{ \pm}-F_{ \pm}}{1-D_{ \pm} F_{ \pm}},
$$

where $D_{ \pm}$and $F_{ \pm}$satisfy the Eilenberger equations in the Riccatti parametrization [10]

$$
\begin{aligned}
& v_{F x} \partial_{x} D_{ \pm}=-\bar{\Delta}_{ \pm}(x)\left(1-D_{ \pm}^{2}\right)+2 \omega_{n} D_{ \pm}, \\
& v_{F x} \partial_{x} F_{ \pm}=-\bar{\Delta}_{ \pm}(x)\left(1-F_{ \pm}^{2}\right)-2 \omega_{n} F_{ \pm},
\end{aligned}
$$

where $v_{F x}$ is the $x$ component of the Fermi velocity, $\omega_{n}=$ $2 \pi T\left(n+\frac{1}{2}\right)$ is the Matsubara frequency, with temperature T. $\bar{\Delta}_{+}(x)\left[\bar{\Delta}_{-}(x)\right]$ is the effective pair potential for leftgoing (right-going) quasiparticles. Since the interface is flat, $F_{ \pm}=-R D_{\mp}$ holds at $x=0$ [10]. Here we consider the situation without mixing different symmetry channels for the pair potential. Then

$$
\bar{\Delta}_{ \pm}(x)=\Delta(x) \Phi_{ \pm}(\theta) \Theta(x)
$$

is satisfied with form factor $\Phi_{ \pm}(\theta)$ given by $1, \cos 2 \theta$, $\pm \sin 2 \theta, \pm \cos \theta$, and $\sin \theta$ for $s, d_{x^{2}-y^{2}}, d_{x y}, p_{x}$, and $p_{y}$-wave superconductors, respectively. The condition in the bulk is $\Delta(\infty)=\Delta$. Since pair potential $\bar{\Delta}_{ \pm}(x)$ is a real number, the resulting $f_{1 \pm}$ is an imaginary number and $f_{2 \pm}$ is a real number.

In the subsequent analysis, we explicitly write $f_{1 \pm}=$ $f_{1 \pm}\left(\omega_{n}, \theta\right)$ and $f_{2 \pm}=f_{2 \pm}\left(\omega_{n}, \theta\right)$. For $x=\infty$, we obtain $f_{1 \pm}\left(\omega_{n}, \theta\right)=0 \quad$ and $\quad f_{2 \pm}\left(\omega_{n}, \theta\right)=\Delta \Phi_{ \pm}(\theta) /$ $\sqrt{\omega_{n}^{2}+\Delta^{2} \Phi_{ \pm}^{2}\left(\theta_{ \pm}\right)}$. Note that $f_{1 \pm}\left(\omega_{n}, \theta\right)$ becomes finite due to the spatial variation of the pair potential and does not exist as the bulk. It follows from Eqs. (2)-(4) that $f_{1 \pm}\left(\omega_{n}, \theta\right)=-f_{1 \pm}\left(-\omega_{n}, \theta\right)$ and $f_{2 \pm}\left(\omega_{n}, \theta\right)=$ $f_{2 \pm}\left(-\omega_{n}, \theta\right)$ for any $x$. It is remarkable that functions $f_{1 \pm}\left(\omega_{n}, \theta\right)$ and $f_{2 \pm}\left(\omega_{n}, \theta\right)$ represent, respectively, oddand even-frequency components of the pair amplitude [7].

Next, we discuss the parity of these pair amplitudes. For an even-parity superconductor $\Phi_{ \pm}(-\theta)=\Phi_{\overline{+}}(\theta)$ while $\Phi_{ \pm}(-\theta)=-\Phi_{\mp}(\theta)$ for an odd-parity one. It follows from Eqs. (2) -(5) that $f_{1 \pm}\left(\omega_{n}, \theta\right)=-f_{1 \mp}\left(\omega_{n},-\theta\right)$ and $f_{2 \pm}\left(\omega_{n}, \theta\right)=f_{2 \mp}\left(\omega_{n},-\theta\right)$ for an even-parity supercon- ductor, and that $f_{1 \pm}\left(\omega_{n}, \theta\right)=f_{1 \mp}\left(\omega_{n},-\theta\right)$ and $f_{2 \pm}\left(\omega_{n}, \theta\right)=-f_{2 \overline{+}}\left(\omega_{n},-\theta\right)$ for an odd-parity superconductor. Note that the parity of the odd-frequency component $f_{1 \pm}\left(\omega_{n}, \theta\right)$ is different from that of the bulk superconductor for all cases.

Let us now focus on the values of the pair amplitudes at interface $x=0$. We concentrate on two extreme cases with (1) $\Phi_{+}(\theta)=\Phi_{-}(\theta)$ and (2) $\Phi_{+}(\theta)=-\Phi_{-}(\theta)$. In the first case, the midgap Andreev resonant state (MARS) is absent since there is no sign change of the pair potential felt by the quasiparticle at the interface. On the other hand, in the second case, MARS is generated near the interface due to the sign change of the pair potential [11]. It is easy to show that $f_{1 \pm}= \pm i(1-R) D_{+} /\left(1+R D_{+}^{2}\right)$ and $f_{2 \pm}=$ $(1+R) D_{+} /\left(1+R D_{+}^{2}\right)$ for case 1 and $f_{1 \pm}=i(1+$ $R) D_{+} /\left(1-R D_{+}^{2}\right)$ and $f_{2 \pm}= \pm(1-R) D_{+} /\left(1-R D_{+}^{2}\right)$ for case 2 . In the low-transparency limit with $T_{m} \rightarrow 0(R \rightarrow$ 1 ), only $f_{2 \pm}$ is nonzero for case 1 and $f_{1 \pm}$ is nonzero for case 2. The remarkable fact is that, in the presence of a sign change of the pair potential, only the odd-frequency pair amplitude exists at the interface for $T_{m} \rightarrow 0$. In general, $f_{1 \pm}$ is pure imaginary and $f_{2 \pm}$ is a real number, so there is a $\pm \pi / 2$ phase shift from interface-induced state.

We next illustrate these results by numerical calculations. We determine the spatial dependence of the pair potential self-consistently [12]. As typical examples, we choose $s$-wave and $p_{x}$-wave pair potentials. In order to understand the angular dependence of the pair amplitude in more detail, we define $\hat{f}_{1}$ and $\hat{f}_{2}$ for $-\pi / 2<\theta<3 \pi / 2$ with $\hat{f}_{1(2)}=f_{1(2)+}(\theta)$ for $-\pi / 2<\theta<\pi / 2$ and $\hat{f}_{1(2)}=$ $f_{1(2)-}(\pi-\theta)$ for $\pi / 2<\theta<3 \pi / 2$. We decompose $\hat{f}_{1(2)}$ into various angular momentum components in the actual calculation. Here, we focus on the lowest angular momentum component. Figure 1 shows $E_{s}(x)$ and $E_{p x}(x)$, which are $s$ - and $p_{x}$-wave components of the even-frequency pair amplitude and the corresponding odd-frequency components $O_{s}(x), O_{p x}(x)$ for $i \omega_{n}=i \pi T$ at $T=0.05 T_{C}$. $T_{C}$ is the transition temperature of the superconductor. As can be seen from Fig. 1(a), in the $s$-wave case the pair potential is suppressed only in the high-transparency regime $(Z=0)$, then odd-frequency component $O_{p x}(x)$ is enhanced near the interface where pair potential $\Delta(x)$ is suppressed. For a low-transparency junction, the magnitude of $O_{p x}(x)$ is negligible. Note that for the $p_{x}$ wave, the magnitude of $O_{s}(x)$ is enhanced near the interface, while $E_{p x}(x)$ is suppressed [see Fig. 1(b)]. For a low-transparency junction where MARS is prominent, the magnitude of $O_{s}(x)$ becomes much larger than the magnitude of $E_{p x}(x)$. The remarkable fact is that the MARS can be reinterpreted as the manifestation of the odd-frequency pair amplitude at the interface.

We next extend the foregoing discussion regarding Eqs. (1)-(5) to the case of bulk odd-frequency pairing in a superconductor. In this case, $\bar{\Delta}_{ \pm}(x)$ depends on a Matsubara frequency $\bar{\Delta}_{ \pm}(x)=\bar{\Delta}_{ \pm}\left(x, \omega_{n}\right)$, with 

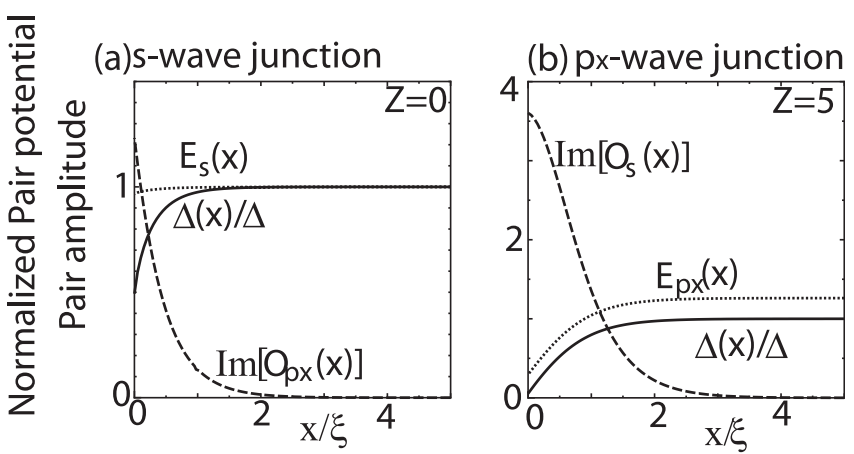

FIG. 1. Spatial dependence of the normalized pair potential (solid line), even-frequency pair amplitudes $E_{s}(x)$ and $E_{p x}(x)$ (dotted lines) and odd-frequency pair amplitudes $O_{s}(x)$ and $O_{p x}(x)$ (dashed lines). (a) $s$-wave bulk state, full-transparency junction with $Z=0$; (b) ETO $p_{x}$-wave bulk state, lowtransparency junction with $Z=5$. Distance $x$ is normalized by $\xi=v_{F} / \Delta$.

$\bar{\Delta}_{ \pm}\left(x, \omega_{n}\right)=-\bar{\Delta}_{ \pm}\left(x,-\omega_{n}\right)$. One can show that $f_{2 \pm}\left(f_{1 \pm}\right)$ becomes an odd-frequency (even-frequency) pair amplitude. In this case, the interface-induced state is an evenfrequency state. Thus, an ETO (ESE) pairing state is induced at the interface for the OTE (OSO) bulk superconductor. Since the pair potential $\bar{\Delta}_{ \pm}(x)$ is an imaginary number, the resulting $f_{1 \pm}$ is a real number and $f_{2 \pm}$ is an imaginary number. In the low-transparency limit, only the odd-frequency state exists at the interface when the sign change of the pair potential is absent. On the other hand, in the presence of the sign change, only an even-frequency state can be realized.

To summarize the above results, we present the relationship between the pair amplitude symmetry in the bulk superconductor and at the interface for $T_{m} \rightarrow 0$ (free surface). As shown in the Table I, there are eight distinct cases which correspond to different combinations of the bulk pairing symmetry and the behavior of the orbital part of the bulk pair potential with respect to reflection from the interface. The pairing symmetry in the diffusive normal metal (DN) attached to the superconductor is also shown. As discussed in Ref. [8], only an $s$-wave even-parity state is possible in the DN. Therefore, when the pair amplitude at the interface has even parity, it can penetrate into DN. However, the odd-parity pair amplitude cannot penetrate into DN. This explains the absence of the proximity effect in the case of the spin-singlet $d_{x y}$-wave pair potential and its presence in the case of the spin-triplet $p_{x}$-wave pair potential [13] as illustrated in Table I, lines 2 and 4.

Let us discuss the Josephson current between evenfrequency $(x<0)$ and odd-frequency superconductors $(x>0)$ to the lowest order in the transmission coefficient $T_{m}$, assuming that spin-flip scattering at the interface is absent. One can calculate the pair amplitude for an $S / N$ system with $S(x<0)$ and $N(x>0)$ by the method described above. We denote the values of the pair amplitudes at the interface as $f_{1 L}, f_{2 L}$ for the left side and $f_{1 R}, f_{2 R}$ for
TABLE I. Relationship between the symmetry of the bulk superconductor and that of the pair amplitude at the interface in the low-transparency limit. The allowed symmetry of the Cooper pair is even-frequency spin-singlet even-parity (ESE), even-frequency spin-triplet odd-parity (ETO), odd-frequency spin-singlet odd-parity (OSO), and odd-frequency spin-triplet even-parity (OTE).

\begin{tabular}{lcccc}
\hline \hline & Bulk state & Sign change & Interface & DN \\
\hline 1 & ESE $\left(s\right.$ or $d_{x^{2}-y^{2}}$ wave $)$ & No & ESE & ESE \\
2 & ESE $\left(d_{x y}\right.$ wave $)$ & Yes & OSO & No \\
3 & ETO $\left(p_{y}\right.$ wave $)$ & No & ETO & No \\
4 & ETO $\left(p_{x}\right.$ wave $)$ & Yes & OTE & OTE \\
5 & OSO $\left(p_{y}\right.$ wave $)$ & No & OSO & No \\
6 & OSO $\left(p_{x}\right.$ wave $)$ & Yes & ESE & ESE \\
7 & OTE $\left(s\right.$ or $d_{\left.x^{2}-y^{2} \text { wave }\right)}$ & No & OTE & OTE \\
8 & OTE $\left(d_{x y}\right.$ wave $)$ & Yes & ETO & No \\
\hline \hline
\end{tabular}

the right side, in the limit $T_{m} \rightarrow 0$. Here, $f_{1 L}, f_{2 R}$ are oddfrequency components and $f_{1 R}, f_{2 L}$ are even-frequency ones. In the presence of a sign change of the pair potential at the interface of the left- (right-)side superconductor, the relations $f_{2 L(R)}=0$ and $f_{1 L(R)} \neq 0$ hold, while in the absence of the sign change of the pair potential, $f_{1 L(R)}=$ 0 and $f_{2 L(R)} \neq 0$. The resulting Josephson current in a superconductor/insulator/superconductor $(S / I / S)$ junction can be represented in the form

$$
R_{N} I(\varphi)=\frac{\pi}{2 e} T \sum_{\omega_{n}} \sum_{\sigma}\left\{\left\langle I_{1}\right\rangle \sin \varphi+\left\langle I_{2}\right\rangle \cos \varphi\right\}
$$

with $I_{1}=f_{1 L} f_{1 R}+f_{2 L} f_{2 R}$ and $I_{2}=f_{1 L} f_{2 R}-f_{1 R} f_{2 L}$, as follows from the boundary condition for the quasiclassical Green's function [14]. The suffix $\sigma$ denotes the direction of the spin. Here $R_{N}$ and $\varphi$ are the resistance of the junction and the macroscopic phase difference between two superconductors. In Eq. (6), the brackets $\langle\ldots\rangle$ denote averaging over the injection angle $\theta$

$$
\left\langle I_{1(2)}(\theta)\right\rangle=\int_{-\pi / 2}^{\pi / 2} d \theta \cos \theta I_{1(2)}(\theta) T_{m} / \int_{-\pi / 2}^{\pi / 2} d \theta \cos \theta T_{m}
$$

According to Table I, there are 16 possible combinations of pairing symmetries in two superconductors. Since the spin structure of Cooper pairs between the left and the right superconductor are different, $I(\varphi)=0$ for the following combinations: 1-7, 1-8, 2-7, 2-8, 3-5, 3-6, 4-5, and 4-6. Also, since the odd- and even-frequency pairing states are realized on both sides of the interface, $I(\varphi)=0$ for the combinations: $1-5,2-6,3-7$, and 4-8. This result is consistent with the previous prediction [2]. The remaining four combinations 1-6, 2-5, 3-8, and 4-7 are worthy of mention. In this case, due to the sign change of the pair potential realized on either the left side or right side at the interface, then the respective pairing symmetry on both sides of the interface is the same, ESE, OSO, ETO, and OTE, as can be seen from Table I. The resulting current-phase relation $I(\varphi)$ is proportional to $\cos \varphi$. This unusual $\varphi$ dependence 
TABLE II. Possible Josephson coupling.

\begin{tabular}{ccccc}
\hline \hline & Type of coupling & Interface & S/I/S & S/DN/S \\
\hline 1 & ESE/OSO & ESE & Yes & Yes \\
2 & ESE/OSO & OSO & Yes & No \\
3 & ETO/OTE & ETO & Yes & No \\
4 & ETO/OTE & OTE & Yes & Yes \\
\hline \hline
\end{tabular}

is relevant to the formation of the broken time reversal symmetry state (BTRSS). It is natural to expect that when the left and the right pair potentials have different parity with respect to the frequency dependence, the BTRSS is formed at this junction. The unusual $\cos \varphi$ current-phase relation stems from the fact that functions $f_{1 L}, f_{1 R}$ have $\pm \pi / 2$ phase shifts from bulk functions $f_{2 L}$ or $f_{2 R}$. Since a macroscopic phase difference $\varphi$ is defined by the phase difference between the bulk functions in the left and right superconductors, the resulting current-phase relation deviates from conventional $\sin \varphi$. The combinations corresponding to nonvanishing Josephson coupling are shown in Table II. If a diffusive normal metal (DN) is placed between the two superconductors, the Josephson current is absent due to impurity scattering, when the interface state has an odd parity.

These results can be applied to actual materials. Fuseya et al. have predicted that the OSO state could be realized in $\mathrm{CeCu}_{2} \mathrm{Si}_{2}$ and $\mathrm{CeRhIn}_{5}$ [6]. This is consistent with some experiments [15]. We propose a robust check of pairing symmetry by using the Josephson effect between ESE (spin-singlet $s$-wave) and OSO superconductors. Here, an ESE superconductor is attached to opposite (parallel) sides of an OSO sample (see Fig. 2). It follows from the above discussion that the induced ESE state of the pair amplitude of the OSO superconductor at the left-side interface and right-side interface have different signs. This additional sign compensates the odd parity of the OSO superconductor, although the ESE state mixes locally near the interface. Then the structure behaves as a $\pi$ junction. The detection of a $\pi$ shift would thus be an unambiguous signature of OSO pairing symmetry, similar to the phase-sensitive tests of $d$-wave symmetry in high- $T_{c}$ cuprates [16].

In summary, we have predicted the lowest-order Josephson coupling between even- and odd-frequency superconductors. Although both superconductors do not break the time reversal symmetry, the resulting current-

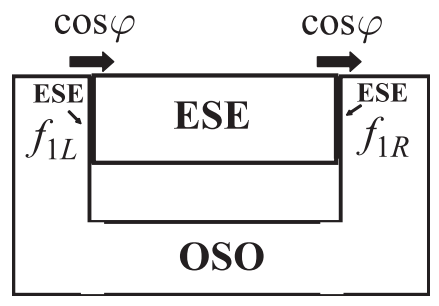

FIG. 2. Schematic illustration of $\pi$-SQUID made of ESE and OSO superconductor junctions. $f_{1 L}=-f_{1 R}$. phase relation is $\cos \varphi$. The underlying physics involves the odd- (even-)frequency pairing state being generated near the interface of the even- (odd-)frequency superconductor. To restore the time reversal invariance in each superconductor, the phase of the interface-induced pair amplitude undergoes a $\pi / 2$ shift from that of the bulk one.

Although we only explicitly studied the $N / S$ junctions, the odd-frequency pairing state can also be expected near impurities and within the Abrikosov vortex cores in evenfrequency superconductors. This implies that an oddfrequency pairing is not at all an exceptional phenomenon as was previously believed but plays a pivotal role in understanding the physics of a nonuniform superconducting system.

This work was supported by a Grant-in-Aid for Scientific Research (Grants No. 17071007, No. 17071005) from the Ministry of Education, Culture, Sports, Science and Technology of Japan and NTT basic research laboratories.

Note added.-After submission of this Letter, we became aware of a reprint by Eschrig [17], who reached similar conclusions about the mixing of even- and oddfrequency pairing states in nonuniform superconductors.

[1] M. Sigrist and K. Ueda, Rev. Mod. Phys. 63, 239 (1991).

[2] E. Abrahams et al., Phys. Rev. B 52, 1271 (1995).

[3] V. L. Berezinskii, JETP Lett. 20, 287 (1974).

[4] A. Balatsky and E. Abrahams, Phys. Rev. B 45, 13125 (1992).

[5] M. Vojta and E. Dagotto, Phys. Rev. B 59, R713 (1999); P. Coleman, E. Miranda, and A. Tsvelik, Phys. Rev. B 49, 8955 (1994).

[6] Y. Fuseya, H. Kohno, and K. Miyake, J. Phys. Soc. Jpn. 72, 2914 (2003).

[7] F. S. Bergeret, A. F. Volkov, and K. B. Efetov, Phys. Rev. Lett. 86, 4096 (2001); F. S. Bergeret, A. F. Volkov, and K. B. Efetov, Rev. Mod. Phys. 77, 1321 (2005).

[8] Y. Tanaka and A. A. Golubov, Phys. Rev. Lett. 98, 037003 (2007).

[9] J. W. Serene and D. Rainer, Phys. Rep. 101, 221 (1983).

[10] M. Eschrig, Phys. Rev. B 61, 9061 (2000).

[11] L. J. Buchholtz and G. Zwicknagl, Phys. Rev. B 23, 5788 (1981); C. R. Hu, Phys. Rev. Lett. 72, 1526 (1994); C. Bruder, Phys. Rev. B 41, 4017 (1990); Y. Tanaka and S. Kashiwaya, Phys. Rev. Lett. 74, 3451 (1995).

[12] Y. Nagato and K. Nagai, Phys. Rev. B 51, 16254 (1995); L. J. Buchholtz et al., J. Low Temp. Phys. 101, 1097 (1995).

[13] Y. Tanaka, Y. V. Nazarov, and S. Kashiwaya, Phys. Rev. Lett. 90, 167003 (2003); Y. Tanaka and S. Kashiwaya, Phys. Rev. B 70, 012507 (2004).

[14] A. V. Zaitsev, Sov. Phys. JETP 59, 1015 (1984).

[15] G. Q. Zheng et al., Phys. Rev. B, 70, 014511 (2004); S. Kawasaki et al., Phys. Rev. Lett. 91, 137001 (2003).

[16] C. C. Tsuei and J.R. Kirtley, Rev. Mod. Phys. 72, 969 (2000).

[17] M. Eschrig et al., J. Low Temp. Phys. 147, 457 (2007). 